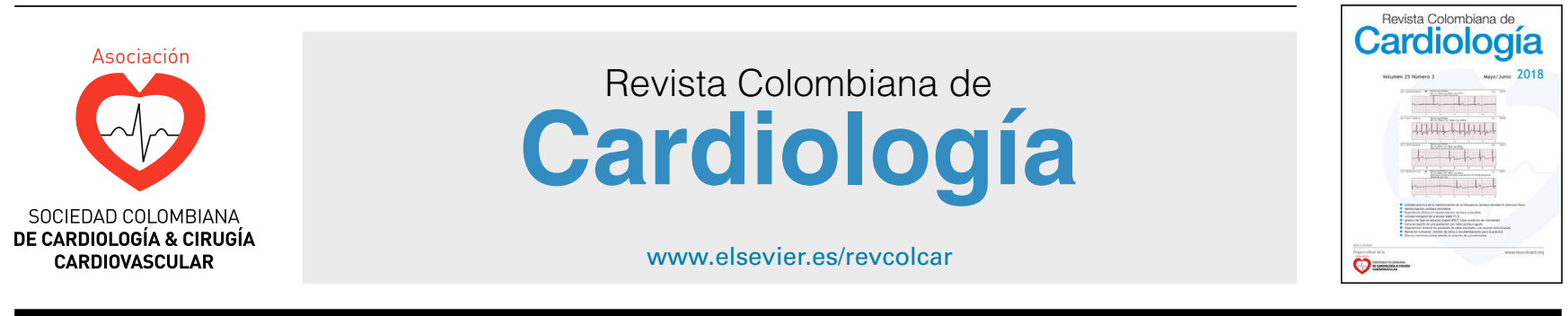

ARTÍCULO ESPECIAL

\title{
Uso terapéutico de los inhibidores de la enzima convertidora de angiotensina en pacientes con COVID-19: las «dos caras de la moneda»
}

\author{
Dora Lucía Vallejo Ardila ${ }^{a, *}$, Yenny Rocío Tamayo Cáceres ${ }^{b}$ y Abraham Alí Munive
}

\author{
a Departamento de Cirugía, Universidad de Melbourne, Facultad de Ciencias de la Salud y Odontológicas. Melbourne, Australia \\ ${ }^{\mathrm{b}}$ Facultad de Salud, Universidad Industrial de Santander, Bucaramanga, Colombia \\ c Fundación Neumológica Colombiana, Bogotá, Colombia
}

Recibido el 13 de junio de 2020; aceptado el 2 de julio de 2020

Disponible en Internet el 16 de julio de 2020

\author{
PALABRAS CLAVE \\ Inhibidores de la \\ Enzima Convertidora \\ de Angiotensina; \\ Enfermedad del \\ coronavirus 2019; \\ Enfermedad \\ cardiovascular; \\ Sistema \\ renina-angiotensina; \\ Respuesta inmune
}

\begin{abstract}
Resumen La evidencia actual es limitada para determinar el impacto del uso de los inhibidores de la enzima convertidora de angiotensina (IECA) en la predisposición al empeoramiento de la enfermedad del coronavirus 2019 (COVID-19). Inicialmente se reportó que en los pacientes con progresión grave de la COVID-19 existía una mortalidad elevada, los cuales tenían antecedentes de hipertensión arterial, diabetes mellitus, enfermedad cardiovascular y enfermedad renal crónica. Parte de estos pacientes también tenía en común que utilizaban IECA, lo cual alertó a la comunidad médica sobre su riesgo potencial en coexistencia con COVID-19. Sin embargo, estudios más recientes de casos-controles encontraron que los inhibidores del sistema reninaangiotensina, incluyendo los IECA, no incrementan el riesgo de COVID-19 o de requerir admisión hospitalaria por esta causa. Diferentes revistas científicas han facilitado el acceso a reportes preliminares, dejando a discreción de la comunidad médica y científica hacer uso de dicha información para promover el desarrollo de estudios que confirmen experimentalmente dichos
\end{abstract}

Abreviaturas: IECA, Inhibidores de la Enzima Convertidora de angiotensina; COVID-19, coronavirus 2019; SARS-CoV-2, el síndrome respiratorio agudo coronavirus 2; HTA, hipertensión arterial; ERA, enfermedad renal aguda; ECV, enfermedad cardiovascular; DB2, diabetes mellitus tipo 2; SRA, sistema renina-angiotensina; AGT, angiotensinógeno; ECA, enzima convertidora de la angiotensina; Ang I, angiotensina I; Ang II, angiotensina II; AT1R, receptores de Ang II tipo 1; AT2R, receptores de Ang II tipo 2; Ang III, angiotensina III; Ang IV, angiotensina IV; Ang-(1-7), angiotensina-(1-7); AT4R, receptor de Ang IV; MasR, receptor de ensamblaje mitocondria; NO, óxido nítrico; ECA 2, isoforma II de la ECA; BRA, bloqueadores del receptor de angiotensina; ARA II, los receptores de la angiotensina II; LPA, lesión pulmonar aguda; SARS-CoV, coronavirus causante del síndrome respiratorio agudo; TMPRSS2, proteasa transmembrana de serina 2; APN, aminopeptidasa NnCoV; DPP4, dipéptidil peptidasa 4; ISRAA, inhibidores del sistema renina-angiotensina-aldosterona; $\mathrm{MHCl}$, mayor de histocompatibilidad I; HLA, complejo de antígeno leucocitario humano; IL, interleucina; VEGF, receptor vascular de crecimiento endotelial; DNT, linfocitos T doble-negativos.

* Autor para correspondencia.

Correos electrónicos: dlucamed@gmail.com, dvallejo@student.unimelb.edu.au (D.L. Vallejo Ardila). 
hallazgos, preclínicos y epidemiológicos, que finalmente impacten en las decisiones de la práctica clínica para beneficiar a los pacientes con COVID-19. En esta revisión de la literatura se exploran los diferentes efectos mediados por los IECA que podrían estar relacionados con la respuesta inmune durante la infección y la transmisión de COVID-19, compilando evidencia disponible que evalúa si en realidad representan un riesgo o si, por el contrario, confieren un efecto protector.

(c) 2020 Sociedad Colombiana de Cardiología y Cirugía Cardiovascular. Publicado por Elsevier España, S.L.U. Este es un artículo Open Access bajo la licencia CC BY-NC-ND (http:// creativecommons.org/licenses/by-nc-nd/4.0/).

\section{KEYWORDS}

Angiotensin

Converting Enzyme

Inhibitors;

COVID-19;

Cardiovascular

disease;

Renin-angiotensin

system;

Immunomodulatory

effect;

Immune response
Therapeutic use of angiotensin converting enzyme inhibitors in patients with COVID-19: the "two sides of the coin"

\begin{abstract}
There is limited evidence for determining the impact of the use of angiotensin converting enzyme inhibitors (ACE-I) in the tendency to worsening of coronavirus-19 disease (COVID-19). It was initially reported that, in patients with serious progression of COVID-19, there was an increased mortality in those that had a history of suffering arterial hypertension, diabetes mellitus, cardiovascular disease, and chronic kidney disease. A proportion of these patients also had in common that they used ACE-I, which alerted the medical community on the potential risk in coexisting with COVID-19. However, in more recent case-control studies, they found that inhibitors of the renin-angiotensin system, including ACE-I, does not increase the risk of COVID-19 or require hospital admission due to this cause. Several scientific journals have provided access to preliminary reports, leaving the use of such information at the discretion of the medical and scientific community for promoting the development of studies that might confirm these preclinical and epidemiological findings experimentally. These may finally have an impact on the clinical practice decisions, in order to benefit patients with COVID-19. In this literature review, the different effects mediated by ACE-I that could be related to the immune response during the infection and transmission of COVID-19 are examined, gathering available evidence that evaluates whether, in reality, they represent a risk or if on the other hand, they confer a protector effect.

(c) 2020 Sociedad Colombiana de Cardiología y Cirugía Cardiovascular. Published by Elsevier España, S.L.U. This is an open access article under the CC BY-NC-ND license (http:// creativecommons.org/licenses/by-nc-nd/4.0/).
\end{abstract}

\section{Introducción}

La evidencia actual es limitada para determinar el impacto del uso como antihipertensivo de los inhibidores de la enzima convertidora de angiotensina (IECA) en la predisposición al empeoramiento de la enfermedad del coronavirus 2019 (COVID-19) ${ }^{1}$, la cual es causada por el virus del síndrome respiratorio agudo coronavirus ${ }^{2}$ (SARS-CoV-2) o nuevo coronavirus (2019-nCoV) ${ }^{2}$. La actual pandemia asociada a COVID-19 ha mostrado una tasa de mortalidad estimada cambiante que se asocia a la edad como principal factor de riesgo y como resultado del síndrome respiratorio agudo ${ }^{3}$. Al respecto, inicialmente se reportó que en los pacientes con progresión grave de la COVID-19 existía una tasa cruda de mortalidad elevada en quienes tenían antecedentes de sufrir de hipertensión arterial (HTA), diabetes mellitus tipo 2 (DB2), enfermedad cardiovascular (ECV) y enfermedad renal crónica $(\mathrm{ERC})^{4}$. Parte de estos pacientes también tenían en común que para el tratamiento de dichas comorbilidades utilizaban IECA, lo cual alertó sobre su riesgo potencial en coexistencia con COVID-19 ${ }^{1}$. La principal variable de confusión a considerar en dichos estudios era el incremento de la prevalencia de estas comorbilidades en la edad avanzada ${ }^{5}$. A pesar de que estos estudios no tomaron en consideración esta y otras variables de confusión que pudieron contribuir a la predicción del riesgo de mortalidad, otros estudios sugieren que es plausible una relación entre el mecanismo fisiopatológico asociado a COVID-19 que pueda estar influenciada por el sistema renina-angiotensinaaldosterona (SRAA) ${ }^{1}$.

Las principales funciones del SRAA son el control de la presión arterial y la homeostasis vascular ${ }^{6}$. Adicionalmente, estudios recientes en el contexto de la respuesta del microambiente tumoral sugieren que el potencial efecto inmunomodulador de los IECA podría actuar de manera directa o indirecta sobre los componentes del eje convencional y alternativo del SRAA ${ }^{7}$. Por lo tanto, el objetivo de esta revisión de la literatura es explorar los diferentes efectos mediados por los IECA que podrían estar relacionados con la respuesta a la infección y transmisión de la COVID-19.

\section{Interacción entre el eje convencional y alternativo del sistema renina-angiotensina}

Los principales componentes del eje convencional del SRAA funcionan como una red intrincada de reacciones 
enzimáticas moduladas por la producción y degradación de péptidos, tales como: angiotensinógeno (AGT), renina, enzima convertidora de la angiotensina (ECA), angiotensina I (Ang I), angiotensina II (Ang II), a través de diversos receptores ${ }^{6}$. Dentro de la cascada de activación del eje convencional se encuentran el receptor de Ang II tipo 1 (AT1R) y el receptor de Ang II tipo $2(A T 2 R)^{6}$. El AGT es producido por el hígado y liberado al torrente sanguíneo. Posteriormente, es hidrolizado por la renina, la cual es producida por las células yuxtaglomerulares del riñón para formar finalmente Ang I. Posteriormente, la Ang I es hidrolizada por la ECA que se encuentra en las células endoteliales pulmonares para producir Ang II. Este es el principal efector del SRA capaz de interactuar con los dos receptores previamente mencionados ${ }^{6}$. Otros péptidos biológicamente activos, como angiotensina III (Ang III) ${ }^{8}$, angiotensina IV (Ang IV)9 y angiotensina-(1-7) (Ang-(1-7))6, se consideran efectores secundarios de menor potencia comparados con Ang II. Por ejemplo, Ang III tiene afinidad por los mismos receptores que Ang II, y al remover el aminoácido arginina de su extremo $\mathrm{N}$-terminal origina Ang IV ${ }^{9}$. Similar a la Ang II, el efecto mediado por la unión de Ang IV a su receptor de Ang IV (AT4R) se inicia en las células endoteliales del pulmón incrementando la liberación de óxido nítrico (NO) ${ }^{9}$. Además, la Ang II estimula la liberación de aldosterona por la corteza de la glándula suprarrenal ${ }^{10}$, y la ECA inactiva la bradiquinina $(\mathrm{BK})^{10}$ (fig. 1).

Por otro lado, el eje alternativo contiene el propéptido Ang-(1-7) que es producto del clivaje de Ang II por la isoforma II de la ECA (ECA2 $)^{11}$. ECA2 es también conocida como homóloga de la ECA o enzima convertidora de angiotensina- carboxipeptidasa ${ }^{11}$. Esta enzima se encuentra principalmente en el endotelio de las arterias coronarias e intrarrenales y el epitelio renal tubular ${ }^{11}$. Asimismo, Ang-(1-7) puede ser producido y metabolizado por la ECA utilizando como substrato angiotensina (1-9) (Ang-(1-9)) y como metabolito inactivo Ang-(1-5), respectivamente. Ang-(1-7) actúa comúnmente a través del receptor de ensamblaje mitocondria (MasR) ${ }^{12}$. También se ha descrito recientemente otro péptido como producto del clivaje Ang-

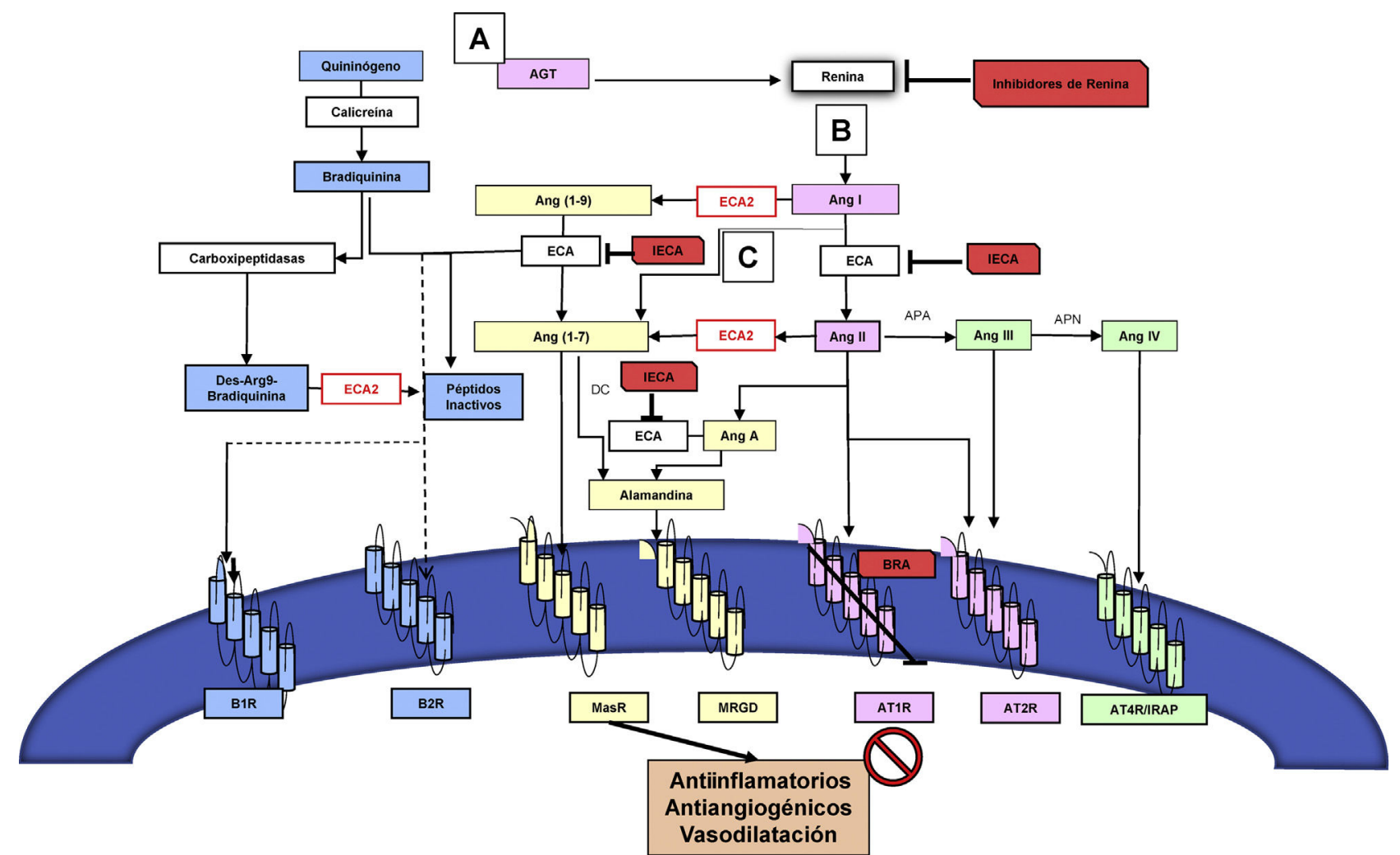

Figura 1 Eje convencional y alternativo del SRA y sus interacciones con el sistema calicreína-cinina. El angiotensinógeno (AGT) es producido y liberado a la circulación por el hígado (A); luego es hidrolizado por la renina, la cual es producida por las células yuxtaglomerulares del riñón (B) para así formar la angiotensina I (Ang I). La Ang I es hidrolizada por la enzima convertidora de angiotensina (ECA) en las células endoteliales de los pulmones $(C)$, para producir finalmente el péptido activo angiotensina II (Ang II). La Ang II interactúa con dos tipos de receptores: receptores de angiotensina II tipo 1 (AT1R) y tipo 2 (AT2R) (en rosado). La enzima convertidora de angiotensina 2 (en rojo) realiza el clivaje de Ang II generando tanto angiotensina-(1-7) (Ang-(1-7), como angiotensina (1-9) (Ang-(1-9). Ang-(1-7) comúnmente actúa vía del receptor de ensamblaje mitocondria (MasR). La alamandina generada como producto del clivaje Ang-(1-7) o angiotensina A (Ang A) tiene afinidad a MasR acoplado a proteína G (MRGD) (en amarillo). El sistema calicreína-cinina es interceptado por ECA y ECA2, los cuales metabolizan la bradicinina y Des-Arg9- Bradiquinina produciendo péptidos inactivos. Se ilustran los receptores de bradicinina tipos 1 (B1R) y 2 (B2R) (en celeste). Niveles de inhibición del SRA por IECA y ARAll ilustrados en rojo. Tanto la inhibición del eje convencional por los IECA/ARAll, como la activación del eje alternativo (Ang-(1-7) /ECA2), promueven efectos antiinflamatorios y antiangiogénicos, y causan vasodilatación. 
(1-7) o angiotensina A (Ang A) conocido como alamandina, el cual tiene afinidad a MasR acoplado a proteína G (MRGD) ${ }^{13}$ (fig. 1).

En general, la activación de cada eje de esta cascada hormonal se puede dar de manera endocrina, paracrina y autocrina, pero es la expresión de los receptores en los órganos diana y la presencia a nivel tisular de dichos componentes la que es capaz de modular la respuesta local a estímulos fisiológicos o fisiopatológicos ${ }^{14}$. Este principio ha sido implementado desde hace más de treinta años para inhibir farmacológicamente en diferentes niveles del eje convencional, utilizando ya sea los IECA ${ }^{15}$, o los bloqueadores del receptor de angiotensina (BRA), específicamente conocidos como antagonistas de los receptores de la angiotensina II (ARA II) ${ }^{16}$ o recientemente con el inhibidor de renina ${ }^{17}$ (fig. 2). Tanto la inhibición del eje convencional por los IECA/ARAll, como la activación del eje alternativo (Ang-(1-7) /ECA2), promueven efectos antiinflamatorios y antiangiogénicos, y causan vasodilatación, lo cual está reflejado en el mejoramiento de la función endotelial, en la prevención de la hipertrofia del miocardio, y, a nivel renal, en el aumento de la tasa de filtración glomerular ${ }^{18}$ (figs. 1 y 2). También se había propuesto modular el SRA utilizando el agente recombinante ECA2, el cual inactiva la Ang II e incrementa la producción de Ang-(1-7) y la estimulación directa del receptor MasR ${ }^{19}$.

\section{La ECA2 y el mecanismo de infección por SARS-CoV-2}

Estudios experimentales que utilizaron modelos animales para investigar acerca de la lesión pulmonar aguda (LPA) y el síndrome de distrés respiratorio agudo (SDRA) descubrieron una marcada reducción en la expresión de $E C A 2^{20}$. Adicionalmente, otro estudio identificó que polimorfismos de inserción/deleción de ECA estaban asociados a susceptibilidad y pronóstico en estas enfermedades ${ }^{21}$. De igual manera, se sabía que la activación de otros componentes de SRA, como Ang II y AT1aR, podían empeorar la presentación del edema pulmonar ${ }^{20}$. Pero para entender el vínculo entre IECA y COVID-19, es necesario comprender, en primer lugar, el mecanismo de infección de este virus utilizando el receptor ECA2, el regulador negativo del SRA (fig. 3), y, en segundo lugar, esclarecer el potencial papel de los IECA en la expresión del receptor ECA2 en los pacientes con COVID-19.

En el 2002, emerge el coronavirus causante del síndrome respiratorio agudo (SARS-CoV) ${ }^{22}$, que comparado con SARS-CoV-2 tiene una tasa de transmisión significativamente menor $^{3}$. Posteriormente, en el año 2005, un estudio in vivo buscaba elucidar el papel de la ECA2, que se expresa en los pulmones de humanos y de ratones, además de las implicaciones de la deficiencia del gen Ace2 en el modelo experimental que simulaba LPA en humanos ${ }^{20}$. Dicha enfermedad se puede presentar en caso de sepsis, broncoaspiración de ácido gástrico, neumonía por infección por SARS-CoV e influenza aviar tipo $\mathrm{A}^{24}$. La base fisiopatológica en la presentación más grave de LPA se caracteriza por edema pulmonar agudo, ocasionado por incremento de la

\section{Efectos terapéuticos beneficiosos de la inhibición del sistema renina-angiotensina}

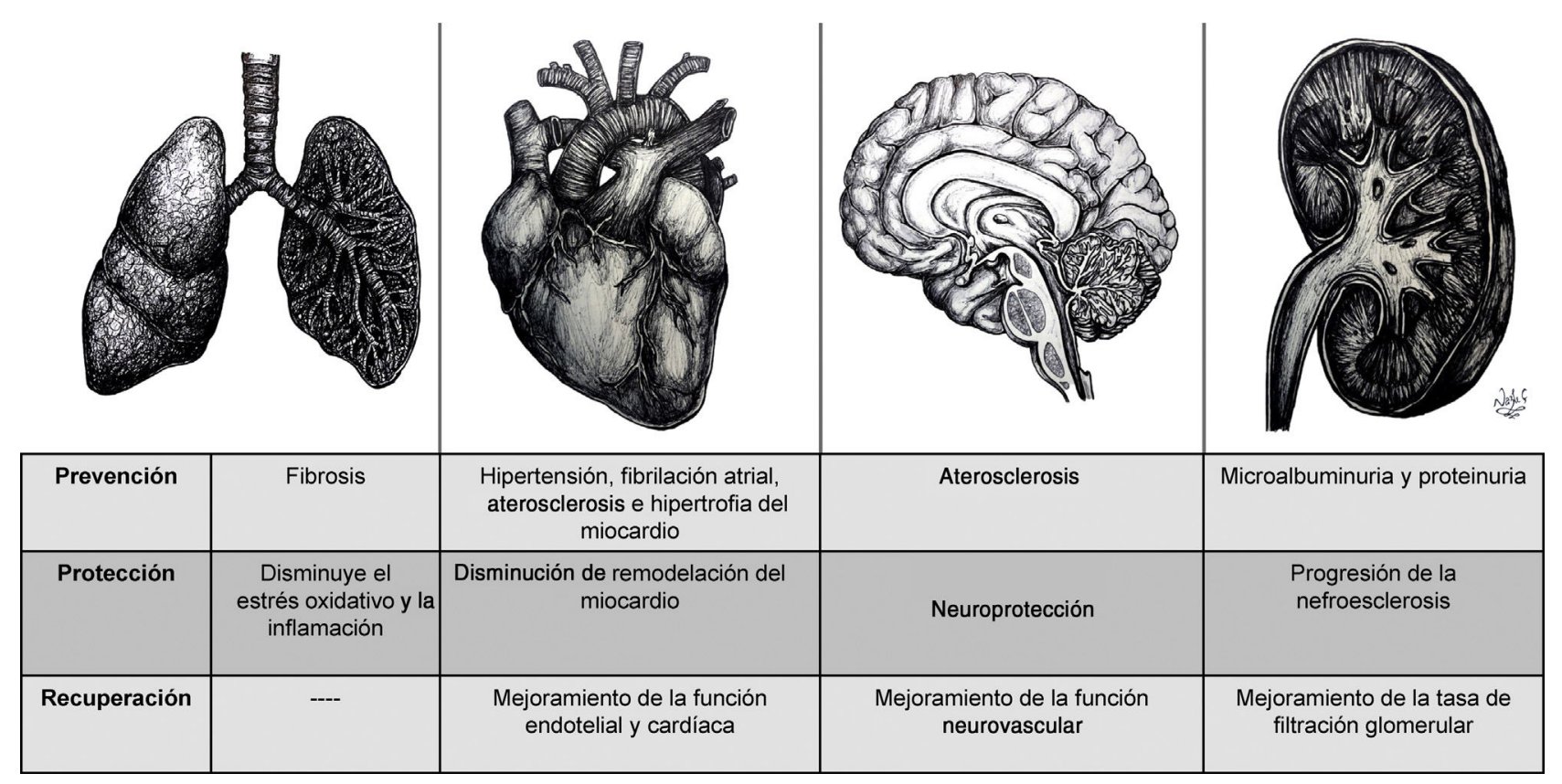

Figura 2 Efecto mediado por los IECA en órganos diana. El efecto pleiotrópico de los IECA genera múltiples beneficios en la escala de prevención, protección y recuperación de órganos diana terapéuticos, tales como pulmón, corazón, cerebro y riñones. 


\section{Efecto protector ECA2 en la lesión pulmonar aguda} Mecanismo de infección de SARS-CoV-2
utilizando el receptor ECA2

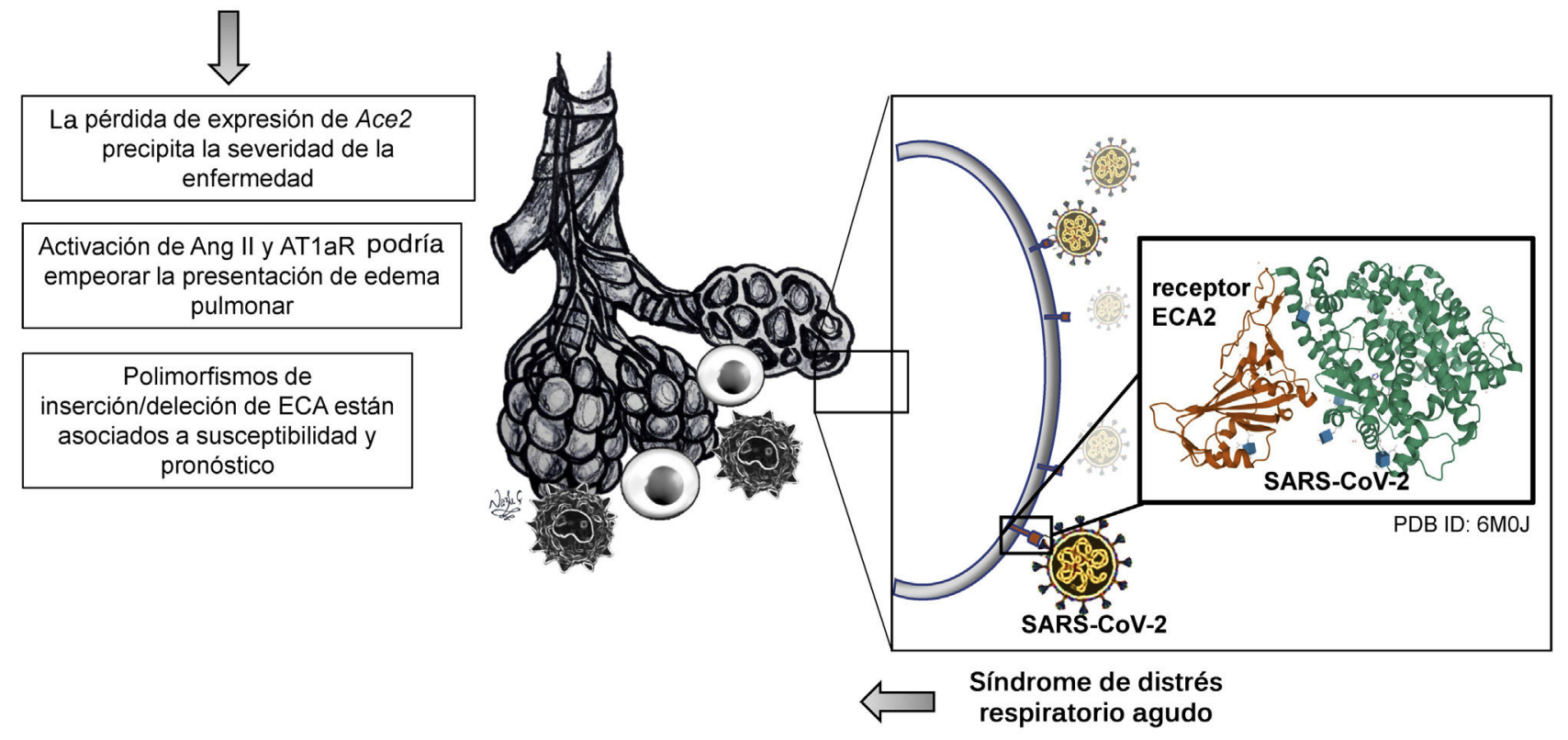

Figura 3 Efecto protector de ECA2 en la lesión pulmonar aguda y como puerta de entrada para SARS-CoV-2. El receptor ECA2 intercepta dos procesos patológicos ejerciendo funciones opuestas.

permeabilidad vascular, aumento de infiltración de células inflamatorias e hipoxia severa ${ }^{24}$. Particularmente, sobre el curso de LPA y el nivel de afectación pulmonar se demostró que la pérdida de expresión de Ace2 precipita la severidad de la enfermedad ${ }^{20}$. Posteriormente, la respuesta a la inoculación por SARS-CoV fue evaluada en el modelo transgénico para Ace2 humano (hAce2), concluyendo que SARS-CoV se replicaba de manera más eficiente en los pulmones modelo transgénico, comparado con el grupo control a los días 3 y 7 , tras la inoculación ${ }^{25}$. Este estudio reportó que los cambios observados en su modelo transgénico replicaban los observados en el SDRA humano, incluyendo hiperemia intersticial, infiltrado de monocitos y linfocitos, presencia de exudados proteicos, y proliferación y descamación del epitelio alveolar ${ }^{25}$.

En tan sólo cuatro meses, se ha descubierto la secuencia genómica del SARS-CoV ${ }^{22}$. Además, se ha caracterizado su origen filogenético y los posibles reservorios naturales ${ }^{23}$. Utilizando modelamiento computacional tridimensional ha sido posible comparar entre la estructura del dominio de unión al receptor de SARS-CoV-2 y SARS-CoV ${ }^{23}$, concluyendo que comparten un $76.5 \%$ de las secuencias de aminoácidos de la glicoproteína S en las espículas (spike), y un alto grado de homología en su secuencia genómica ${ }^{26}$. Previamente, estudios de cristalografía y de interacción bioquímica habían demostrado que la glicoproteína $S$ en las espículas (spike) de SARS-CoV tiene una gran afinidad por el receptor ECA2 ${ }^{26}$. Para resumir, la replicación y la transmisión de célula a célula se da por la unión de la glicoproteína $S$ en las espículas (spike) de SARS-CoV-2 al receptor ECA2, seguido por el clivaje proteolítico de la ECA2 por la proteasa transmembrana de serina 2 (TMPRSS2) ${ }^{27}$ (fig. 3).

Ahora bien, reportes recientes especificaron que el dominio de unión a receptor de SARS-CoV-2 reconocido por el receptor ECA2 corresponde al residuo 394 (glutamina), e inclusive es más eficiente dicha afinidad comparada con SARS-CoV, lo cual incrementa la capacidad de SARS-CoV-2 para transmitirse ${ }^{28}$. Con el fin de confirmar si SARS-CoV-2 utilizaba a su vez receptor de entrada de ECA2, se realizaron estudios de infectividad viral in vitro utilizando células HeLa que expresaban el receptor ECA2 comparadas con aquellas que no. Este estudio indicó que, en el caso de humanos, murciélago horseshoe (de la China), civeta y cerdos, SARS-CoV-2 es capaz de utilizar ECA2 como receptor de entrada, como también se reportó que no utiliza otro tipo de receptores de coronavirus, tales como aminopeptidasa $\mathrm{NnCoV}$ (APN) y dipéptidil peptidasa 4 (DPP4) ${ }^{2}$. El reporte preliminar del perfil de expresión de la ECA2 en tejido pulmonar humano utilizando single-cell RNA seq identificó una alta concentración de este receptor en las células alveolares tipo II, lo cual sugiere un tropismo particular por SARS-CoV-2 ${ }^{29}$. Sin embargo, dicho estudio descubrió que esta población celular expresaba enriquecimiento en genes que favorecen la replicación viral ${ }^{29}$. Anteriormente se había determinado el nivel normal de expresión tisular de ECA2 predominantemente en ovario, intestino, riñón, corazón y testículos ${ }^{30}$ (fig. 4).

Finalmente, las hipótesis sobre el mecanismo fisiopatológico de infección de SARS-CoV-2, el uso terapéutico de los IECA y el potencial daño que podrían inducir en pacientes con COVID-19 han surgido, en gran parte, como extrapolación de resultados obtenidos en las investigaciones sobre SARS-CoV aplicados a COVID-1931, las cuales sugieren que el incremento de la actividad del eje convencional ECA/Ang II en relación con el eje alternativo ECA2/Ang-(1-7) puede promover LPA en COVID-1932. 

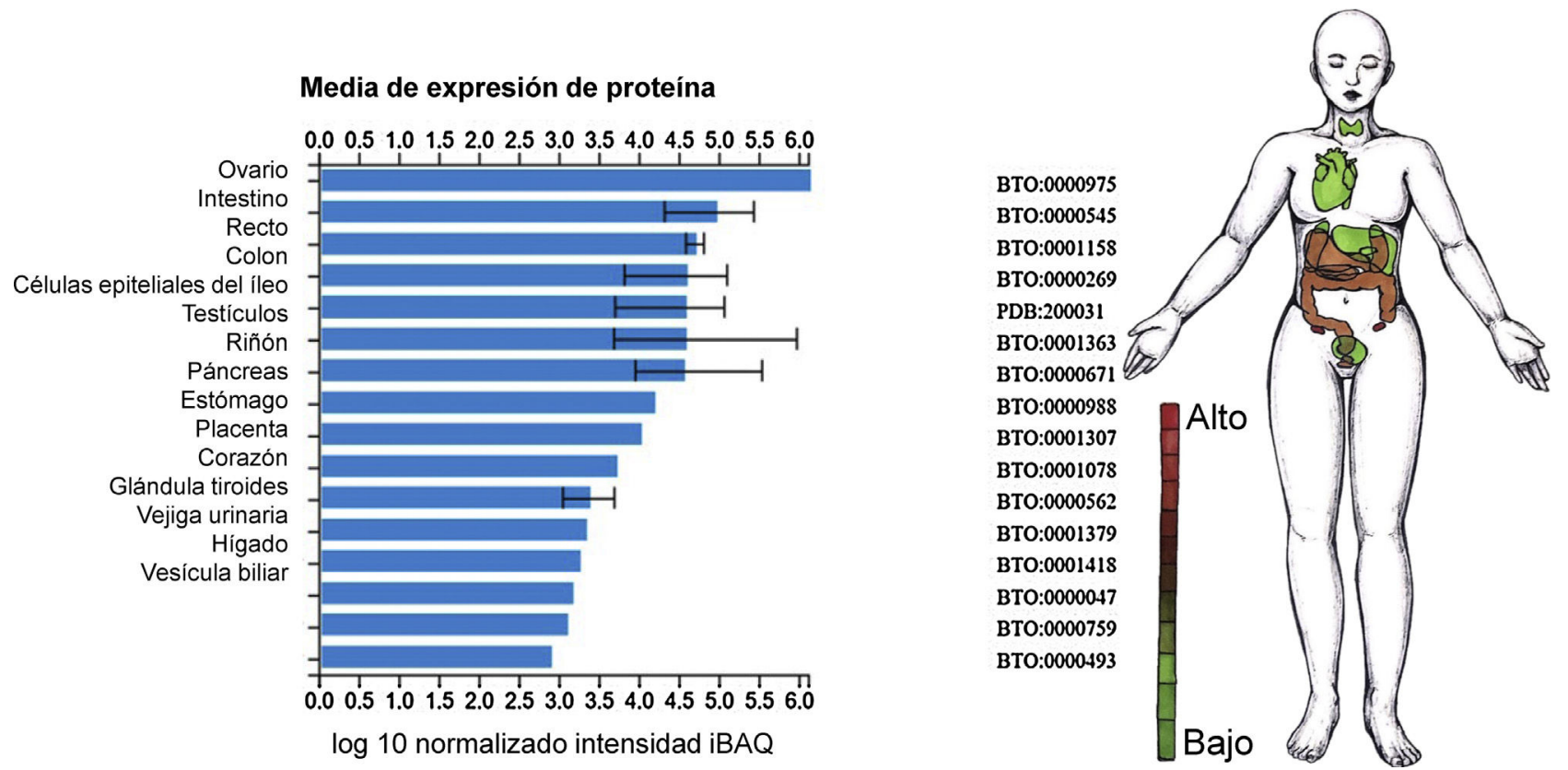

Figura 4 Nivel de expresión de ECA2 en tejidos y líneas celulares. Estudios de proteómica integrada (ProteomicDB) ${ }^{30}$ reportan un nivel de expresión tisular de ECA2 (Q9BYF1) predominante en ovario, intestino, testículo, riñón, páncreas, estómago, placenta, corazón, glándula tiroides, vejiga urinaria, hígado y vesícula biliar.

\section{Uso terapéutico de los IECA en pacientes con COVID-19}

En la actualidad, los IECA continúan siendo el grupo de medicamentos antihipertensivos más utilizados por millones de pacientes en todo el mundo ${ }^{33}$. Sin embargo, el uso terapéutico de esta medicación en pacientes que cursan con COVID-19 se ha tornado controversial ${ }^{1,32}$, a causa de evidencia contradictoria basada en las hipótesis sobre el papel de la ECA2 como puerta de entrada y replicación viral de SARS-CoV- $2^{31}$, y en los hallazgos de estudios observacionales que reportaron un aumento en la severidad de infección por SARS-CoV-2 en pacientes con múltiples comorbilidades, que adicionalmente usaban IECA ${ }^{4}$ (tabla suplementaria 1).

En primer lugar, la hipótesis que supone el uso de los IECA como deletéreo en pacientes con COVID-19 sugiere que SARS-CoV-2 ingresa a través del receptor ECA2, lo cual en presencia de los IECA puede incrementar los niveles de ECA2, promoviendo de esta manera la entrada y replicación viral ${ }^{28}$. Desde el punto de vista fisiológico, la supresión de la ECA2 acontece como parte del proceso de internalización del receptor desde la superficie celular, lo cual disminuye tanto la presencia tisular de ECA2 como la generación de Ang-(17). Por ende, esto incrementa los niveles de Ang II, y así mismo la Ang-(1-7) se convierte en sustrato disponible para la ECA convirtiéndolo en un péptido biológicamente menos activo $^{12}$. En consecuencia, la activación del eje ECA/Ang II desencadena una respuesta inflamatoria mediada por AT1R, e incrementa la severidad de LPA $^{32}$, así como es posible exista un incremento en la expresión y la actividad de ECA2, en órganos como riñón y corazón, que finalmente incremente el riesgo de susceptibilidad a la infección por
SARS-CoV-2 en pacientes que utilizan regularmente IECA ${ }^{32}$ (fig. $5 \mathrm{~A}$ ).

En segundo lugar, la hipótesis opuesta presume que Ang II como efector principal del SRA desencadena la activación AT1R induciendo vasoconstricción, angiogénesis e inflamación. Por lo tanto, el uso de IECA estaría indicado para contrarrestar dichos efectos en LPA, lo que al mismo tiempo podría contribuir al incremento de Ang-(1-7) por ECA2, y la activación de MasR $^{12}$. De lo anterior, es preciso aclarar que los estudios disponibles no han esclarecido el efecto de los IECA en la expresión y actividad ECA2: además, no hay evidencia suficiente sobre sus implicaciones en el funcionamiento del tejido pulmonar ${ }^{25}$ (fig. 5B).

En tercer lugar, está la hipótesis sobre la relación directa entre la alta prevalencia de HTA en pacientes con COVID19, quienes habían presentado un curso de enfermedad agravado. Series de caso provenientes de China indicaron una prevalencia estimada del $15 \%^{34}$ de HTA entre los pacientes con COVID-19 (tabla suplementaria 1$)^{5}$. El hecho que estos pacientes mostraran un cuadro de enfermedad más severo que requería soporte ventilatorio, o que incluso morían, alertó a la comunidad médica sobre el riesgo potencial de esta condición coexistente, así como del uso de los IECA ${ }^{5}$. A pesar de que estos reportes no hicieron el ajuste riguroso de predicción de riesgo considerando variables de confusión, como la edad avanzada, ya se había demostrado en otras enfermedades infecciosas, que comorbilidades como la HTA son un factor determinante en el pronóstico ${ }^{5}$. Ahora, en cuanto al uso de IECA, en esta serie de casos se estimó que sólo entre un 25 a 30\% de los pacientes con HTA fueron tratados con IECA ${ }^{35}$. Otras comorbilidades, ECV y ERC5, fueron reportadas en pacientes con COVID-19 y podrían estar relacionadas, a su vez, con el uso de IECA puesto que estarían indicados en caso de presentar falla 


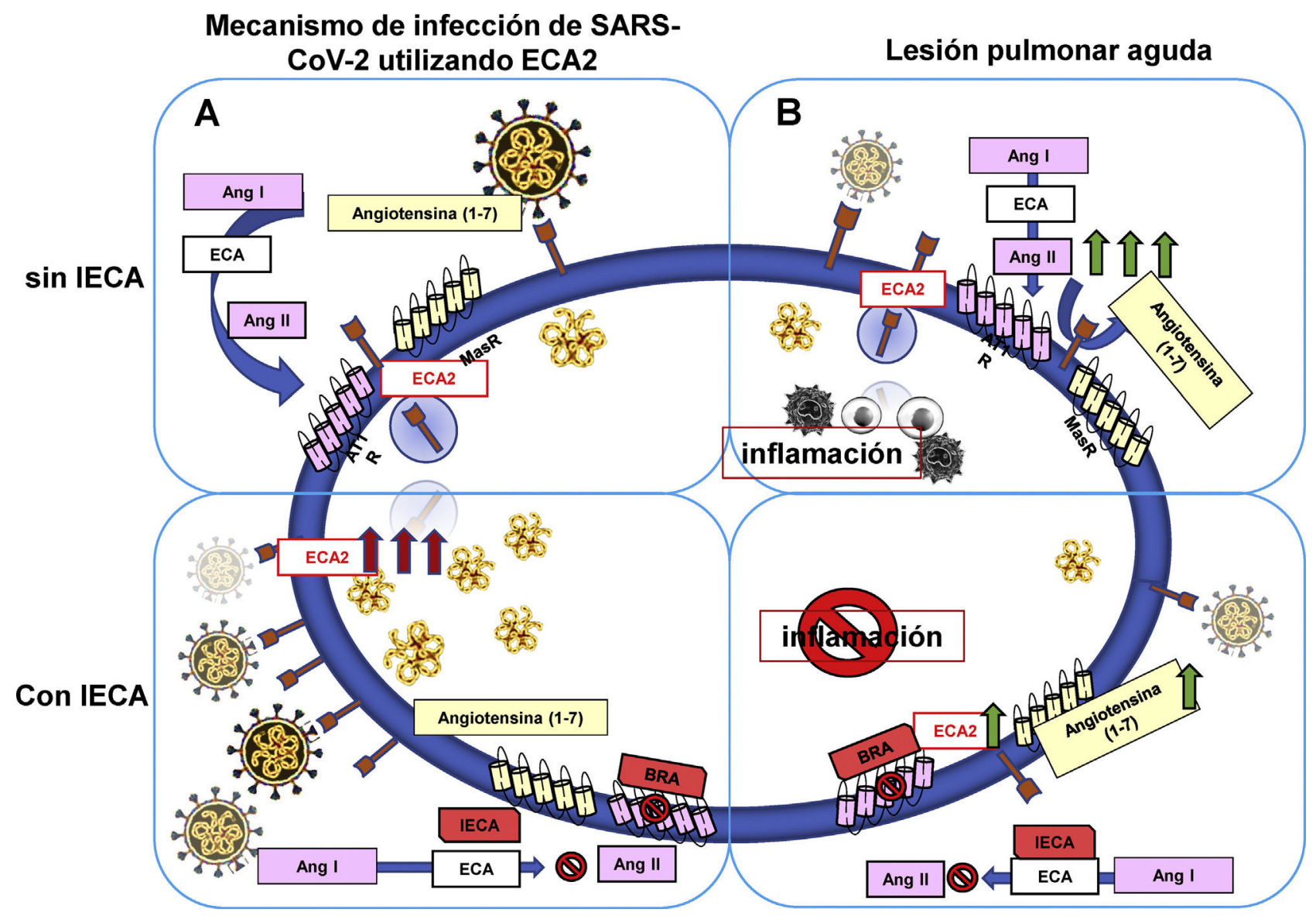

Figura 5 Uso terapéutico de los IECA en pacientes con COVID-19. Hipótesis (A): el uso de IECA en pacientes con COVID-19 aumenta la expresión del receptor ECA2 en la superficie celular; por ende, incrementa la replicación y transmisión viral. Hipótesis (B): la activación de angiotensina II (Ang II) y del receptor de angiotensina II tipo1 (AT1R) promueve la severidad de la lesión pulmonar aguda y la inflamación, la cual disminuye al adicionar IECA/ARAll. Esto promueve la generación de angiotensina (1-7) [Ang (1-7)] por la ECA2, lo cual finalmente disminuye la inflamación.

cardíaca con impedimento funcional de ventrículo izquierdo y proteinuria severa, respectivamente. Tomando en consideración lo expuesto se puede concluir que el nivel de evidencia es insuficiente, y que, por lo tanto, decisiones sobre el manejo médico de pacientes con COVID-19, que sufren de hipertensión, ECV y ERC, y, al mismo tiempo, reciben IECA, requieren de un mejor soporte científico ${ }^{1,5}$. A pesar de esto, las diferentes sociedades científicas y médicas emitieron comunicados en los que no recomiendan la suspensión de los IECA en pacientes con COVID-19 por riesgo de descompensación de las comorbilidades tratadas, y, además, porque podrían prevenir lesiones cardiovasculares y reducir el daño pulmonar causado por COVID-1936.

En vista de la falta de estudios de base poblacional, en marzo del año en curso se realizó el primer estudio de casos y controles que investigó la asociación entre requerir admisión hospitalaria por COVID-19 y el uso de inhibidores del sistema renina-angiotensina-aldosterona (ISRAA) comparado con el uso de otros medicamentos antihipertensivos. Dicho estudio incluyó 1.139 casos y 10 controles por cada caso, los cuales fueron elegidos de manera aleatoria de la Base de datos para la Investigación Farmacoepidemiológica en Atención Primaria (BIFAP) de España (hasta el año 2018), y emparejados individualmente por edad, sexo, región y fecha de ingreso hospitalario. Este estudio concluye que, en el contexto de COVID-19, no se observó que ninguno de los ISRAA, IECA o ARAll aumenten el riesgo de necesitar ingreso hospitalario, incluyendo los casos fatales y aquellos que requirieron ingreso a unidades de cuidado intensivo. Esta asociación se mantuvo al ajustar por variables de confusión tales como edad, género y riesgo de enfermedad cardiovascular. Por el contrario, se observó que el uso de ISRAA se asoció con la reducción significativa en el riesgo para la necesidad de ingreso hospitalario en pacientes diabéticos con COVID-1937. Los hallazgos de este estudio fueron corroborados por otro más reciente, de base poblacional de casos-controles realizado en la región de Lombardía (Italia), incluyendo 6.272 casos confirmados de COVID-19 emparejados con 30.759 individuos beneficiarios del sistema regional de salud. Los resultados no mostraron ninguna asociación entre el uso de IECA (razón de disparidad (OR) ajustado, 0,95 [IC95\%:0,86 - 1,05]) o ARAll (OR ajustado 0,96 [IC95\%:0,87 - 1,07]) con riesgo de infección por COVID-1938, así como tampoco se encontró ninguna asociación entre IECA y casos con enfermedad de COVID-19 severa o casos fatales (OR ajustado 0,83 $[\text { IC95\%:0,63- 1,10]) })^{38}$. 


\section{Efectos de los IECA podrían reprogramar la respuesta inmune en COVID-19}

Aún es limitada la evidencia disponible sobre cómo se realiza el proceso de presentación de antígeno y la activación de linfocitos $T$ específicos en la respuesta viral de SARS-CoV-2 ${ }^{39}$, lo cual depende del complejo mayor de histocompatibilidad I [MHCl o complejo del antígeno leucocitario humano (HLA) ${ }^{40}$. Previamente se había demostrado que diferentes polimorfismos de HLA podrían conferir riesgo o ser protectores en la infección por influenza A (H1N1) y SARS-CoV ${ }^{41}$. En cuanto a la inmunidad celular a SARS-CoV2 , se reportó que la proporción tanto de linfocitos $\mathrm{T} \mathrm{CD4}^{+}$ como de $\mathrm{CD}^{+}$circulantes se encuentra reducida, pero en estatus de activación expresando HLA-DR y CD38, similar a los hallazgos de fase aguda en SARS-CoV ${ }^{42}$. Se supo que la memoria inmunológica basada en la respuesta de célula $T$ específica permanece hasta seis años después de la infección por SARS-CoV en 14 de 23 pacientes ${ }^{43}$. El perfil de citocinas en COVID-19 se caracteriza por incremento en interleucina (IL)-2, IL-7, factor estimulante de granulocitos e interferón gamma inducible, entre otros ${ }^{44}$.

En un estudio retrospectivo de pacientes de Wuhan, se reportó que la ferritina elevada (promedio de $1.129 .6 \mathrm{ng} / \mathrm{ml}$ no sobreviviente versus $614.0 \mathrm{ng} / \mathrm{ml}$ sobrevivientes) e IL6 (promedio de $6.8 \mathrm{ng} / \mathrm{ml}$ sobreviviente versus $11.4 \mathrm{ng} / \mathrm{ml}$ no sobrevivientes), y la proporción neutrófilo-leucocito elevada podría incrementar el riesgo de mortalidad inducida por hiperinflamación ${ }^{45}$.

El papel regulador del SRA en varios procesos patológicos, tales como inflamación ${ }^{25}$ y proliferación tumoral, ha sido investigado en modelos animales ${ }^{7,46}$, contrario al efecto modulador del sistema inmune de $\operatorname{los} \mathrm{IECA}^{7,46}$, el cual no ha sido ampliamente explorado y podría ser el punto de partida para nuevas propuestas de investigación en COVID$19^{47}$. Entre los efectos de los IECA que podrían reprogramar la respuesta inmune y que, además, se podrían extrapolar desde el campo de la inmunoterapia para el cáncer con el fin de desarrollar nuevas terapias para COVID-19 se encuentran la disminución de hipoxia y acidosis tisular, la regulación de la cascada inflamatoria, la disminución del estrés oxidativo ${ }^{7}$ y el efecto modulador de los linfocitos $\mathrm{T}^{46}$ (fig. 6).

La hipoxia y la acidosis tumoral inducen una cascada de factores transcripcionales, factores de crecimiento tumoral y citocinas proinflamatorias, como el factor inducible por hipoxia $(\mathrm{FIH}-1)$, el factor de crecimiento de transformación beta (TGF- $\beta$ ) y la IL- 6 , los cuales generan inmunosupresión local $^{7}$. Los IECA impiden que se genere el estímulo inicial disminuyendo la hipoxia tisular e impidiendo la producción de Ang II. El estrés oxidativo, que impide el funcionamiento de linfocitos T, también es un efecto de la Ang II/AT1R, el cual puede ser contrarregulado selectivamente con ARAII ${ }^{48}$.

Recientemente se descubrió que los IECA producen un cambio dinámico temporal en subpoblaciones específicas de linfocitos $\mathrm{T}$ en el parénquima hepático peritumoral y en las metástasis hepáticas inducidas en el modelo murino de cáncer colorrectal, incrementando tanto la población de linfocitos $\mathrm{CD}^{+}$y $\mathrm{CD}^{+}$, como disminuyendo significativamente ${ }^{46}$ los linfocitos $\mathrm{CD4}^{+}$(fig. 6). Asimismo, reportes iniciales que examinaron el conteo absoluto de linfocitos $T$ en pacientes con COVID-19, quienes habían recibido inhibidores del SRA (IECA/BRA), mostraron un conteo más elevado de linfocitos $\mathrm{CD}^{+}$y $\mathrm{CD} 8^{+}$, mientras no se observaron cambios significativos en linfocitos $\mathrm{CD}^{+}$en sangre periférica, comparados con aquellos que recibieron otros medicamentos antihipertensivos ${ }^{49}$. Adicionalmente, este estudio indicó una tendencia hacia la disminución de los niveles de IL-6 en sangre periférica en pacientes con COVID-19 que recibieron IECA/BRA comparado con el

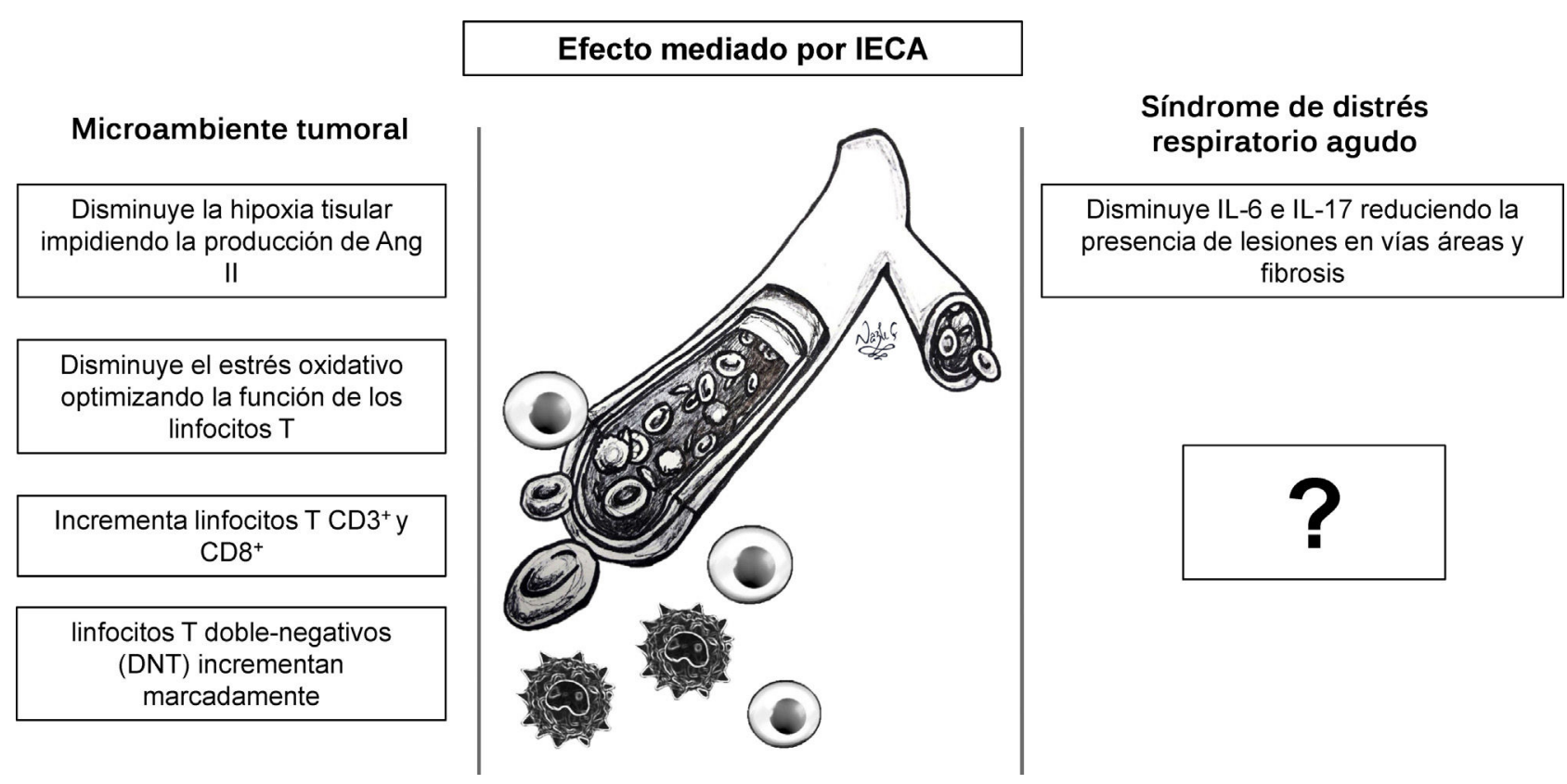

Figura 6 El efecto mediado por los IECA reprograma la respuesta inmune en el microambiente tumoral. Estudios sobre el efecto inmunomodulador de los IECA en la respuesta antitumoral puede ser el punto de encuentro entre inmunoterapias para cáncer y nuevas estrategias terapéuticas para COVID-19. 
grupo control ${ }^{49}$. Previamente estudios realizados en un modelo murino de obliteración de vías aéreas mostraron que después de administrar $(10 \mathrm{mg} / \mathrm{kg} /$ día $)$ de IECA/ARAII por 5 días, la IL-6 y la IL-17 disminuyeron marcadamente reduciendo la presencia de lesiones en vías áreas y fibrosis $^{50}$. Finalmente, se ha descrito que los linfocitos $T$ doble-negativos (DNT) incrementan de manera significativa en respuesta a IECA ${ }^{46}$. Pese a que representan un porcentaje minúsculo (1-3\%) de individuos saludables, se observó un marcado incremento en la respuesta a la influenza A, lo que demuestra su papel como regulador de la respuesta inmune en el parénquima pulmonar ${ }^{51}$. Ahora bien, ante la falta de información sobre la respuesta inmune humoral y celular se hace imperativo continuar investigando para comprender cómo se presenta el síndrome de liberación de citocinas en COVID-19, y si la administración de un agente bloqueador de IL-6, como tocilizumab o siltuximab, podría tener un efecto beneficioso en estos pacientes ${ }^{52}$.

\section{Perspectivas e implicaciones clínicas}

Sin duda, existe una relación entre el SRA y la COVID-19. En esta revisión de la literatura se exploraron algunos de los aspectos más relevantes sobre la ECA2 y el mecanismo de infección por SARS-CoV-2, controversias sobre el uso terapéutico de los IECA en pacientes con COVID-19 y los efectos de los IECA que podrían reprogramar la respuesta inmune en COVID-19.

Hoy día, las investigaciones son insuficientes para determinar con certeza si los estudios preclínicos sobre el aumento en la expresión de ECA2 causado por los IECA representan un riesgo en pacientes con COVID-19, o si, por el contrario, los IECA confieren efectos protectores en el manejo de la LPA. Al respecto, estudios más recientes de casos-controles encontraron que los inhibidores del SRAA, incluyendo los IECA, no incrementan el riesgo de COVID19 o de requerir admisión hospitalaria por esta causa ${ }^{37,38}$. En la actualidad se desarrollan ensayos clínicos para evaluar la eficacia y la seguridad de los IECA en pacientes con COVID-19 (ClinicalTrials.gov identificador: NCT04311177 y NCT04312009) (tabla suplementaria 1).

Para concluir, es preciso señalar que desde el inicio de la pandemia ocasionada por COVID-19, un sinnúmero de revistas científicas han facilitado el acceso a resultados preliminares, que en muchos casos no han sido revisados por pares, dejando a discreción de la comunidad científica hacer uso de dichos resultados de manera responsable, para promover estudios que confirmen, de manera experimental, hallazgos preclínicos y epidemiológicos, que finalmente impacten en las decisiones de la práctica clínica para beneficiar al paciente. Aun se requieren ensayos clínicos prospectivos para investigar si el uso de IECA puede reducir la incidencia y la mortalidad asociada a la COVID-19 o a la LPA, tanto en pacientes con o sin indicación terapéutica de uso de IECA.

\section{Financiación}

Este trabajo es apoyado por el Departamento de Ciencia, Tecnología e Innovación (COLCIENCIAS- Colombia) Programa de Doctorados en el exterior 679 y la Universidad de
Melbourne, Facultad de Medicina, odontología y ciencias de la Salud (Programa de becas 2018-2021), Australia.

\section{Agradecimientos}

Este trabajo es apoyado por el Departamento de Ciencia, Tecnología e Innovación (COLCIENCIAS- Colombia), Programa de Doctorados en el exterior 679 y la Universidad de Melbourne, Facultad de Medicina, odontología y ciencias de la Salud (Programa de becas 2018-2021). Un agradecimiento especial a José Leonardo Vallejo Ardila por la edición de la versión de artículo en español, y a Nazli Calik por las ilustraciones de los órganos.

\section{Anexo. Material adicional}

Se puede consultar material adicional a este artículo en su versión electrónica disponible en doi:10.1016/j.rccar. 2020.07.001.

\section{Bibliografía}

1. Danser AHJ, Epstein M, Batlle D. Renin-angiotensin system blockers and the COVID-19 Pandemic: at present there is no evidence to abandon renin-angiotensin system blockers. Hypertension. 2020;75:1382-5. Disponible en https://doi.org/10.1161/hypertensionaha.120.15082.

2. Wu C, Chen X, Cai Y, Xia Ja, Zhou X, Xu S, et al. Risk Factors Associated With Acute Respiratory Distress Syndrome and Death in Patients With Coronavirus Disease 2019 Pneumonia in Wuhan, China. JAMA Internal Medicine. 2020. https://doi.org/10.1001/jamainternmed.2020.0994.

3. Verity R, Okell LC, Dorigatti I, Winskill P, Whittaker C, Imai N, et al. Estimates of the severity of coronavirus disease 2019: a model-based analysis. The Lancet Infectious Diseases. 2020. Disponible en https://doi.org/https://doi.org/10.1016/S14733099(20)30243-7.

4. Zhou F, Yu T, Du R, Fan G, Liu Y, Liu Z, et al. Clinical course and risk factors for mortality of adult inpatients with COVID-19 in Wuhan. China: a retrospective cohort study. Lancet. 2020;395:1054-62. Disponible en https://doi.org/10.1016/s0140-6736(20)30566-3.

5. Vaduganathan M, Vardeny O, Michel T, McMurray JJV, Pfeffer MA, Solomon SD. Renin-angiotensin-aldosterone system inhibitors in patients with COVID-19. N Eng J Med. 2020. Disponible en https://doi.org/10.1056/NEJMsr 2005760.

6. Carey RM, Siragy HM. Newly Recognized Components of the Renin-Angiotensin System: Potential Roles in Cardiovascular and Renal Regulation. Endocrine Reviews. 2003;24:261-71, http://dx.doi.org/10.1210/er.2003-0001.

7. Vallejo-Ardila DL, Fifis T, Burrell LM, Walsh K, Christophi C. Renin-angiotensin inhibitors reprogram tumor immune microenvironment: A comprehensive view of the influences on anti-tumor immunity. Oncotarget. 2018;9:35500-11. Disponible en https://doi.org/10.18632/oncotarget.26174.

8. Moeller I, Allen AM, Chai SY, Zhuo J, Mendelsohn FA. Bioactive angiotensin peptides. J Hum Hypertens. 1998;12:289-93. Disponible en https://doi.org/10.1038/sj.jhh.1000640.

9. Mustafa T, Lee JH, Chai SY, Albiston AL, McDowall SG, Mendelsohn FA. Bioactive angiotensin peptides: focus on angiotensin IV. J Renin Angiotensin Aldosterone Syst. 2001;2:205-10. Disponible en https://doi.org/10.3317/jraas.2001.032. 
10. Bader M, Ganten D. Update on tissue renin-angiotensin systems. J Mol Med (Berl). 2008;86:615-21. Disponible en https://doi.org/10.1007/s00109-008-0336-0.

11. Donoghue M, Hsieh F, Baronas E, Godbout K, Gosselin M, Stagliano N, et al. A novel angiotensin-converting enzymerelated carboxypeptidase (ACE2) converts angiotensin I to angiotensin 1-9. Circ Res. 2000;87:E1-9. Disponible en https://doi.org/10.1161/01.res.87.5.e1.

12. Santos RA, Simoes e Silva AC, Maric C, Silva DM, Machado RP, de Buhr I, et al. Angiotensin-(1-7) is an endogenous ligand for the $G$ protein-coupled receptor Mas. Proc Natl Acad Sci U S A. 2003;100:8258-63. Disponible en https://doi.org/10.1073/pnas.1432869100.

13. Lautner RQ, Villela DC, Fraga-Silva RA, Silva N, VeranoBraga T, Costa-Fraga F, et al. Discovery and characterization of alamandine: a novel component of the reninangiotensin system. Circ Res. 2013;112:1104-11. Disponible en https://doi.org/10.1161/circresaha.113.301077.

14. Lavoie JL, Sigmund CD. Minireview: overview of the renin-angiotensin system-an endocrine and paracrine system. Endocrinology. 2003;144:2179-83. Disponible en https: //doi.org/10.1210/en.2003-0150.

15. Erdos EG. Conversion of angiotensin I to angiotensin II. Am J Med. 1976;60:749-59. Disponible en https://doi.org/10.1016/0002-9343(76)90889-5.

16. Bhardwaj G. How the antihypertensive losartan was discovered. Expert Opin Drug Discov. 2006;1:609-18. Disponible en https://doi.org/10.1517/17460441.1.6.609.

17. Muller DN, Luft FC. Direct renin inhibition with aliskiren in hypertension and target organ damage. Clin J Am Soc Nephrol. 2006;1:221-8. Disponible en https://doi.org/10.2215/cjn.01201005.

18. Iwanami J, Mogi $M$, Iwai $M$, Horiuchi $M$. Inhibition of the renin-angiotensin system and target organ protection. Hypertens Res. 2009;32:229-37. Disponible en https://doi.org/10.1038/hr.2009.5.

19. Ferreira AJ, Bader M, Santos RA. Therapeutic targeting of the angiotensin-converting enzyme 2/Angiotensin-(17)/Mas cascade in the renin-angiotensin system: a patent review. Expert Opin Ther Pat. 2012;22:567-74. Disponible en https: / / doi.org/10.1517/13543776.2012.682572.

20. Imai Y, Kuba K, Rao S, Huan Y, Guo F, Guan B, et al. Angiotensin-converting enzyme 2 protects from severe acute lung failure. Nature. 2005;436:112-6. Disponible en https://doi.org/10.1038/nature03712.

21. Marshall RP, Webb S, Bellingan GJ, Montgomery HE, Chaudhari B, McAnulty RJ, et al. Angiotensin converting enzyme insertion/deletion polymorphism is associated with susceptibility and outcome in acute respiratory distress syndrome. Am J Respir Crit Care Med. 2002;166:646-50. Disponible en https://doi.org/10.1164/rccm.2108086.

22. Rota PA, Oberste MS, Monroe SS, Nix WA, Campagnoli R, Icenogle JP, et al. Characterization of a novel coronavirus associated with severe acute respiratory syndrome. Science. 2003;300:1394-9. Disponible en https://doi.org/10.1126/science.1085952.

23. Wan Y, Shang J, Graham R, Baric RS, Li F. Receptor Recognition by the Novel Coronavirus from Wuhan: an Analysis Based on Decade-Long Structural Studies of SARS Coronavirus. Journal of Virology. 2020;94:e00127-00120. Disponible en https://doi.org/10.1128/jvi.00127-20.

24. Ware LB, Matthay MA. The acute respiratory distress syndrome. N Engl J Med. 2000;342:49-1334. Disponible en https://doi.org/10.1056/nejm200005043421806.

25. Yang $X H$, Deng $W$, Tong Z, Liu YX, Zhang LF, Zhu H, et al. Mice transgenic for human angiotensin-converting enzyme 2 provide a model for SARS coronavirus infection. Comp Med. 2007;57:9-450.
26. Li F, Li W, Farzan M, Harrison SC. Structure of SARS coronavirus spike receptor-binding domain complexed with receptor. Science. 2005;309:1864-8. Disponible en https://doi.org/10.1126/science.1116480.

27. Hoffmann M, Kleine-Weber H, Schroeder S, Krüger N, Herrler T, Erichsen S, et al. SARS-CoV-2 Cell Entry Depends on ACE2 and TMPRSS2 and Is Blocked by a Clinically Proven Protease Inhibitor. Cell. 2020;181:271-80, e278. Disponible en https://doi.org/https://doi.org/10.1016/j.cell.2020.02. 052.

28. Lan J, Ge J, Yu J, Shan S, Zhou H, Fan S, et al. Structure of the SARS-CoV-2 spike receptor-binding domain bound to the ACE2 receptor. Nature. 2020. Disponible en https: / / doi.org/10.1038/s41586-020-2180-5.

29. Zou X, Chen K, Zou J, Han P, Hao J, Han Z. Singlecell RNA-seq data analysis on the receptor ACE2 expression reveals the potential risk of different human organs vulnerable to 2019-nCoV infection. Front Med. 2020. Disponible en https: / / doi.org/10.1007/s11684-020-0754-0.

30. Uhlén M, Fagerberg L, Hallström BM, Lindskog C, Oksvold P, Mardinoglu A, et al. Proteomics. Tissue-based map of the human proteome. Science. 2015;347:1260419. Disponible en https://doi.org/10.1126/science.1260419.

31. Kuba K, Imai Y, Rao S, Gao H, Guo F, Guan B, et al. A crucial role of angiotensin converting enzyme 2 (ACE2) in SARS coronavirusinduced lung injury. Nat Med. 2005;11:9-875. Disponible en https://doi.org/10.1038/nm1267.

32. South AM, Tomlinson L, Edmonston D, Hiremath S, Sparks MA. Controversies of renin-angiotensin system inhibition during the COVID-19 pandemic. Nature Reviews Nephrology. 2020. Disponible en https://doi.org/10.1038/s41581-020-0279-4.

33. Chen YJ, Li LJ, Tang WL, Song JY, Qiu R, Li Q, et al. First-line drugs inhibiting the renin angiotensin system versus other first-line antihypertensive drug classes for hypertension. Cochrane Database of Systematic Reviews. 2018. Disponible en https://doi.org/10.1002/14651858.CD008170.pub3.

34. Guan WJ, Ni Z-Y, Hu Y, Liang W-H, Ou C-Q, He J-X, et al. Clinical Characteristics of Coronavirus Disease 2019 in China. N Eng J Med. 2020. Disponible en https://doi.org/10.1056/NEJMoa2002032.

35. Lu J, Lu Y, Wang X, Li X, Linderman GC, Wu C, et al. Prevalence, awareness, treatment, and control of hypertension in China: data from 1.7 million adults in a population-based screening study (China PEACE Million Persons Project. Lancet. 2017;390:2549-58. Disponible en https://doi.org/10.1016/s0140-6736(17)32478-9.

36. Guo J, Huang Z, Lin L, Lv J. Coronavirus Disease 2019 (COVID-19) and Cardiovascular Disease: A Viewpoint on the Potential Influence of Angiotensin-Converting Enzyme Inhibitors/Angiotensin Receptor Blockers on Onset and Severity of Severe Acute Respiratory Syndrome Coronavirus 2 Infection. J Am Heart Assoc. 2020;9:e016219. Disponible en https://doi.org/10.1161/jaha.120.016219.

37. de Abajo FJ, Rodríguez-Martín S, Lerma V, Mejía-Abril G, Aguilar M, García-Luque A, et al. Use of renin-angiotensin-aldosterone system inhibitors and risk of COVID-19 requiring admission to hospital: a case-population study. Lancet. 2020;395:1705-14. Disponible en https: / / doi.org/10.1016/s0140-6736(20)31030-8.

38. Mancia G, Rea F, Ludergnani M, Apolone G, Corrao G. Renin-Angiotensin-Aldosterone System Blockers and the Risk of Covid-19. N Eng J Med. 2020;382:40-2431. Disponible en https://doi.org/10.1056/NEJMoa2006923.

39. Shi Y, Wang Y, Shao C, Huang J, Gan J, Huang X, et al. COVID-19 infection: the perspectives on immune responses. Cell Death \& Differentiation. 2020. Disponible en https: / / doi.org/10.1038/s41418-020-0530-3.

40. Nguyen A, David JK, Maden SK, Wood MA, Weeder BR, Nellore $A$, et al. Human leukocyte antigen susceptibility map for SARS- 
CoV-2. medRxiv. 2020, 2020.2003.2022.20040600. Disponible en https: / /doi.org/10.1101/2020.03.22.20040600.

41. Lin M, Tseng H-K, Trejaut JA, Lee H-L, Loo J-H, Chu C-C, et al. Association of HLA class I with severe acute respiratory syndrome coronavirus infection. BMC Medical Genetics. 2003;4:9. Disponible en https://doi.org/10.1186/1471-2350-4-9.

42. Thevarajan I, Nguyen THO, Koutsakos M, Druce J, Caly L, van de Sandt CE, et al. Breadth of concomitant immune responses prior to patient recovery: a case report of nonsevere COVID-19. Nature Medicine. 2020;26:453-5. Disponible en https: / / doi.org/10.1038/s41591-020-0819-2.

43. Tang F, Quan Y, Xin Z-T, Wrammert J, Ma M-J, Lv H, et al. Lack of Peripheral Memory B Cell Responses in Recovered Patients with Severe Acute Respiratory Syndrome: A Six-Year Follow-Up Study. J Immunol. 2011;186:7264-8. Disponible en https://doi.org/10.4049/jimmunol.0903490.

44. Huang C, Wang Y, Li X, Ren L, Zhao J, Hu Y, et al. Clinical features of patients infected with 2019 novel coronavirus in Wuhan. China. Lancet. 2020;395:497-506. Disponible en https://doi.org/10.1016/s0140-6736(20)30183-5.

45. Ruan Q, Yang K, Wang W, Jiang L, Song J. Clinical predictors of mortality due to COVID-19 based on an analysis of data of 150 patients from Wuhan. China. Intensive Care Med. 2020. Disponible en https://doi.org/10.1007/s00134-020-05991-x.

46. Vallejo Ardila DL, Walsh K, Fifis T, Paolini R, Georgios K, Christophi C, et al. Immunomodulatory effects of renin-angiotensin system inhibitors on $\mathrm{T}$ lymphocytes in mice with colorectal liver metastases. Journal of ImmunoTherapy of Cancer. 2020;0:e000487. Disponible en https://doi.org/10.1136/jitc-2019-000487.

47. Bernstein KE, Khan Z, Giani JF, Cao D-Y, Bernstein EA, Shen XZ. Angiotensin-converting enzyme in innate and adaptive immunity. Nature Reviews Nephrology. 2018;14:25-36. Disponible en https://doi.org/10.1038/nrneph.2018.15.

48. Rudemiller NP, Crowley SD. Interactions between the immune and the renin-angiotensin systems in hypertension.
Hypertension (Dallas, Tex: 1979). 2016;68:289-96. Disponible en https://doi.org/10.1161/HYPERTENSIONAHA.116.06591.

49. Meng J, Xiao G, Zhang J, He X, Ou M, Bi J, et al. Renin-angiotensin system inhibitors improve the clinical outcomes of COVID-19 patients with hypertension. Emerg Microbes Infect. 2020;9:757-60. Disponible en https: / / doi.org/10.1080/22221751.2020.1746200.

50. Weber J, Tiriveedhi V, Takenaka M, Lu W, Hachem R, Trulock $\mathrm{E}$, et al. Inhibition of renin angiotensin aldosterone system causes abrogation of obliterative airways disease through inhibition of tumor necrosis factor-alpha-dependant interleukin- 17. J Heart Lung Transplant. 2012;31:419-26. Disponible en https://doi.org/10.1016/j.healun.2011.12.012.

51. Neyt K, GeurtsvanKessel CH, Lambrecht BN. Double-negative $T$ resident memory cells of the lung react to influenza virus infection via CD11c(hi) dendritic cells. Mucosal Immunol. 2016;9:999-1014, http://dx.doi.org/10.1038/mi.2015.91.

52. Liu B, Li M, Zhou Z, Guan X, Xiang Y. Can we use interleukin-6 (IL-6) blockade for coronavirus disease 2019 (COVID-19)-induced cytokine release syndrome (CRS)? Journal of Autoimmunity. 2020;111. Disponible en 102452-102452. https://doi.org/10.1016/j.jaut.2020.102452. 